\title{
An Inverse Method to Reconstruct Complete Stiffness Information of Rubber Bushing
}

\author{
Gang Lei, ${ }^{1}$ Qian Chen, ${ }^{2}$ Ying Liu, ${ }^{2}$ and Jingjing Jiang ${ }^{2}$ \\ ${ }^{1}$ Key Laboratory of Advanced Manufacturing Technology for Automobile Parts, Chongqing University of Technology, \\ Ministry of Education, Chongqing 400054, China \\ ${ }^{2}$ Faculty of Mechanical Engineering, Chongqing University of Technology, Chongqing 400054, China
}

Correspondence should be addressed to Gang Lei; ganglei4786@126.com

Received 7 July 2013; Accepted 23 August 2013

Academic Editor: Xing Chen

Copyright (C) 2013 Gang Lei et al. This is an open access article distributed under the Creative Commons Attribution License, which permits unrestricted use, distribution, and reproduction in any medium, provided the original work is properly cited.

\begin{abstract}
A specific rubber bushing, with only radial and axial stiffness data having been acquired, is studied. In terms of the hyperelastic material of this bushing, three-term Ogden law is utilized as the material constitutive model which requires to be characterized. Without the material mechanical tests provided, a parameter identification method is proposed for searching a group of acceptable parameters which are able to model rubber-like material of this rubber bushing. In this case, based on the nonlinear finite element analysis method and optimization technique, the parameters of material law are determined, and the rotational stiffness of this bushing is also evaluated. The complete stiffness information has been established.
\end{abstract}

\section{Introduction}

Rubber is recognized as an engineering material since the vulcanization of rubber being pioneered by Charles Goodyear in 1839 [1]. Unlike metals which require relatively few properties to characterize their mechanical behavior, the rubber-like materials present more complex with nonlinearity in terms of both geometric and material behavior. The traditional method to characterize rubber-like materials constitutive laws is evaluated through the sets of experimental data of different types of deformation. Uniaxial tension, planar tension, and biaxial tension tests are as the recommendations to identify the model parameters. Charlton et al. [1] utilized synthetic test data to characterize the mechanical behavior of rubber via FEA. Chen et al. [2] proposed a tension model for analyzing the rubber layers based on the theory of elasticity. Suphadon and Busfield [3] proposed a demanding test at solving a large strain elasticity problem of a rubber cylinder subjected to a combination of tension and torsion based on the applicable FE method. The validity of different stored energy functions was also studied. The image analysis is also used in material parameter of rubber; see [4]. However, obtaining the rubber-like material behavior without material experimental information due to the shortage of necessary experimental appliances also needs to be considered.

Several methods are proposed at solving problems of parameter identification. Baker and Shrot [5] calculated material parameters from descriptors which involve inverse parameter identification methods without finite element simulations. Florentin and Lubineau [6] have explained how an appropriate use of the constitutive equation gap method can help in verification of material model parameters.

In this paper, an inverse finite element method for parameters identification is introduced, which involves the stiffness information of a specific component. More specifically, we focus on the parameters identification for one of the material constitutive laws and the evaluation for rotational stiffness of one certain rubber bushing.

\section{Constitutive Laws}

Generally, vulcanized rubber is assumed as an incompressible hyperelastic material [7]. Hyperelastic material is described as a "strain energy potential," which defines the strain energy stored in the material per unit of reference volume as a function of the deformation at that point in the material. The most 
common constitutive laws for modeling the hyperelastic materials behavior are the Mooney [8], Rivlin [9], Twizell and Ogden [10], and Yeoh [11] models. Each constitutive model is a special form of the strain energy potential [12].

Li and Yang [13] have pointed out that Ogden, Yeoh, and Martins models are the best ones that fit the siliconerubber experimental data. Considering these hyperelastic models, the model whose parameter determination is the most complicated is the Ogden model [10] due to its relatively large number of parameters [14].

The Ogden strain energy potential is expressed in terms of the principal stretches $\left(\lambda_{1}, \lambda_{2}\right.$, and $\left.\lambda_{3}\right)$. Thus, the constitutive equation of Ogden takes the form $[1,15]$

$$
U=\sum_{i=1}^{N} \frac{2 \mu_{i}}{\alpha_{i}^{2}}\left(\lambda_{1}^{\alpha_{1}}+\lambda_{2}^{\alpha_{1}}+\lambda_{3}^{\alpha_{1}}-3\right)+\sum_{i=1}^{N} \frac{1}{D_{i}}(J-1-R)^{2 i},
$$

where $\mu_{i}, \alpha_{i}$ are material constants, $N$ is the number of terms in the function, the stretch ratios $\lambda_{1}, \lambda_{2}$, and $\lambda_{3}$ are eigenvalues of the deformation tensor, $J$ is the ratio to the deformed volume to the undeformed volume, and $D_{i}$ defines the material compressibility (when $D_{i}=0$, the material is considered completely incompressible). An ideal incompressible material maintains constant volume:

$$
j=1, \quad R=0 .
$$

The Ogden model, (1), then becomes

$$
U=\sum_{i=1}^{N} \frac{2 \mu_{i}}{\alpha_{i}^{2}}\left(\lambda_{1}^{\alpha_{1}}+\lambda_{2}^{\alpha_{1}}+\lambda_{3}^{\alpha_{1}}-3\right)
$$

Note that as required by the definition of a strain energy potential, Ogden's strain energy vanishes when the material is in its natural, undeformed state [16]:

$$
\lambda_{1}=\lambda_{2}=\lambda_{3}=1
$$

Ogden constitutive law is considered one of the most successful functions in describing the large deformation range of rubber-like materials. In this paper, therefore, the constitutive equation of three-term Ogden, with six coefficients, is utilized to define the property of this rubber material.

\section{Parameter Identification for Rubber-Like Material of Rubber Bushing}

In this paper, based on the combination of the nonlinear FE method and optimization technique, the parameters of hyperelastic material will be defined to describe the mechanical characteristics of rubber bushing. Meanwhile, the complete stiffness information about rubber bushing can be obtained.

During the identification process, two FE simulations are performed to replicate the radial and axial stiffness tests. The parameters of the material constitutive law are determined to fit the experimental result. Firstly, a set of initial values are assigned to the parameters of constitutive law. Simulate the translational stiffness of rubber bushing described by

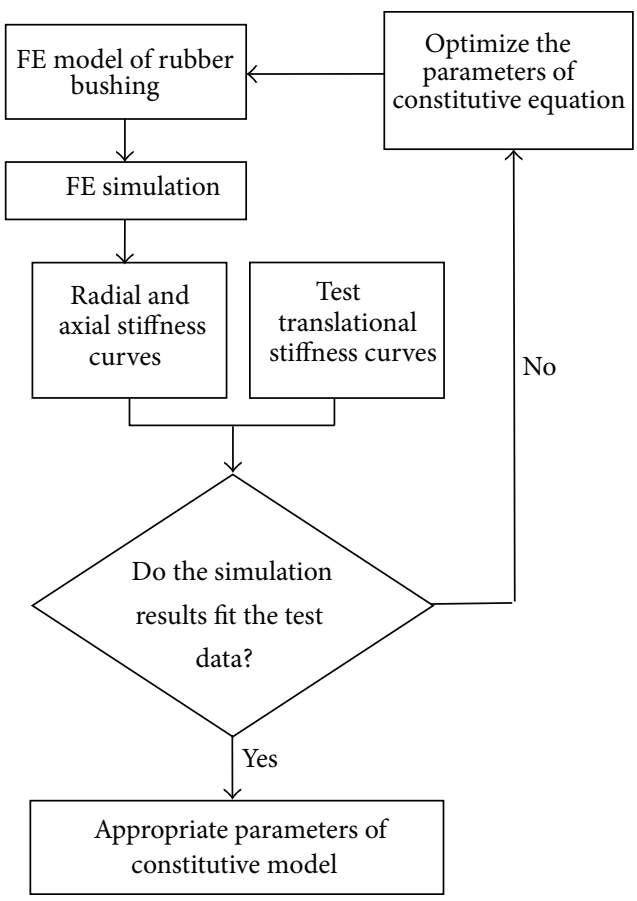

FIGURE 1: Typical steps in parameters identification cycle.

the foregoing parameters. If this result fails to fit the experimental data, an optimization procedure needs to be conducted to find the appropriate parameters which are able to model an effective hyperelastic material achieving to describe the mechanical characteristics of rubber bushing. Figure 1 demonstrates the main steps in parameters identification cycle.

3.1. Translational Stiffness Tests. The axial and radial stiffness tests were conducted due to the axisymmetrical structure of rubber bushing and limited test equipment. As shown in Figure 2, the axial stiffness is along $x$ direction, and the radial stiffness is along $y$ or $z$ direction.

The translational stiffness tests provide axial and radial stiffness information which will be utilized as the standard data in the next parameter identification process.

3.2. Translational Stiffness Simulations. In this work, a CAD model of rubber bushing is imported to the FE software. This model is meshed to finite elements, material properties and appropriate boundary conditions are set subsequently to acquire a completely rubber bushing FE model. To simulate the radial and axial stiffness data, the software ABAQUS is used. This process is the preparation for the next stageparameter identification.

More specifically, two load steps are set as the boundary conditions to fully assess the models fit. In the first step, a forced displacement was applied on the outer surface of bushing simultaneously intermediate shaft fixed. This is a preloaded step for fixing the bushing and establishing nonlinear contact steadily. In the second step, the loads were applied on the pipe around the center hole, incrementally from $0.0 \mathrm{~N}$ 


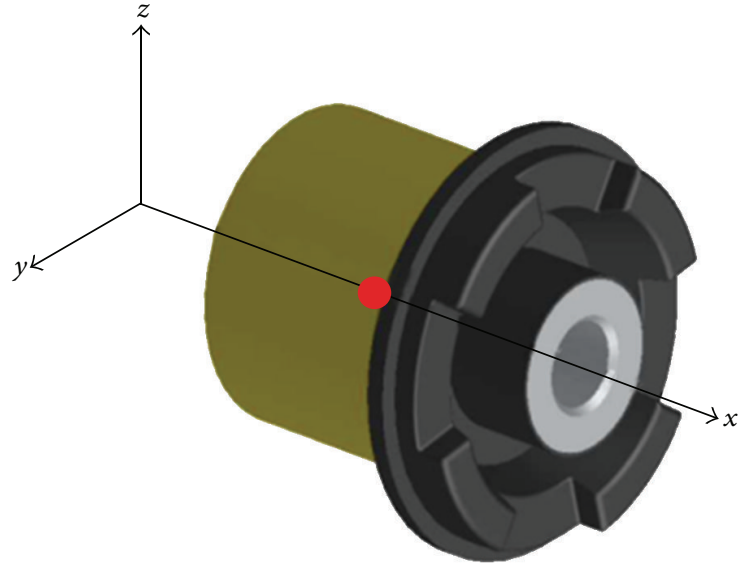

FIGURE 2: CAD model of rubber bushing.

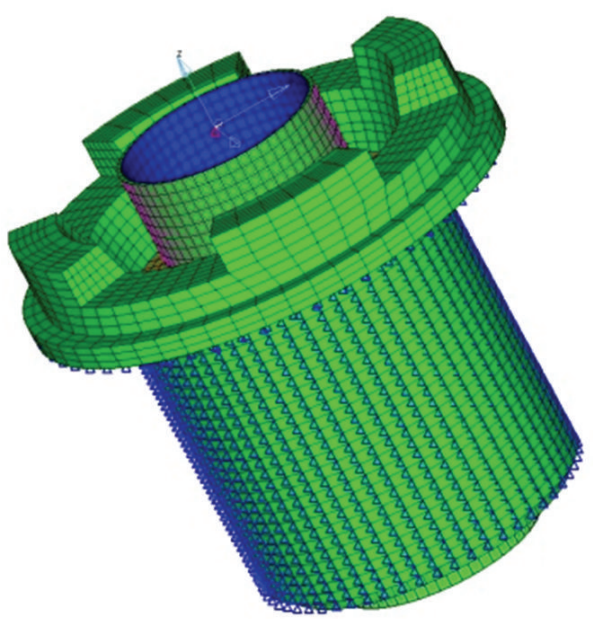

FIgURE 3: FE model of rubber bushing.

to $1000 \mathrm{~N}$ in $x$ direction and $0.0 \mathrm{~N}$ to $3000 \mathrm{~N}$ in $y$ direction which enables the axial stiffness and the radial stiffness to be evaluated by the FE software, respectively. The three-term Ogden law is applied as constitutive model for this rubber bushing, and a set of initial guesses are defined:

$$
\begin{gathered}
\mu_{1}=1, \quad \mu_{2}=0.00029, \quad \mu_{3}=5 e-06, \\
\alpha_{1}=1.1, \quad \alpha_{2}=11.5, \quad \alpha_{3}=-10 .
\end{gathered}
$$

Figure 3 shows the FE model of rubber bushing with completely boundary conditions and material information.

The simulations were carried out for the two test described earlier: a radial stiffness test and an axial stiffness test. The simulated versus experimental displacement-stress curvilinear relationship is shown in Figure 4. Comparatively, there is a considerable gap between two curves which represent the stiffness steamed from the test and simulation, respectively. It is apparent that the initial coefficients of Ogden constitutive model do not suit this rubber bushing,
TABLE 1: The values of design variables.

\begin{tabular}{lcc}
\hline Design variables & Lower bound & Upper bound \\
\hline$\mu_{1}=1.0$ & 0.5 & 2.0 \\
$\mu_{2}=2.9 e-04$ & $2.0 e-04$ & $3.8 e-04$ \\
$\mu_{3}=5 e-06$ & $4 e-06$ & $6 e-06$ \\
$\alpha_{1}=1.1$ & 0.6 & 1.6 \\
$\alpha_{2}=11.5$ & 10 & 13 \\
$\alpha_{3}=-10.0$ & -5 & -15 \\
\hline
\end{tabular}

and parameter identification is needed to find accurate coefficients of material constitutive model consequently.

3.3. Parameter Identification. Parameters of the constitutive equation of rubber are determined based on the combination of translational stiffness test data and finite element optimization techniques. When the results of simulation present apparent deviations from test data, the coefficients of the constitutive model should be redefined. This process can be completed by optimization software.

The six coefficients $\left(\mu_{1}, \mu_{2}, \mu_{3}, \alpha_{1}, \alpha_{2}\right.$, and $\left.\alpha_{3}\right)$ in the threeterm Ogden strain energy model are selected as the design variables, since all their values influence stiffness of rubber bushing. Based on experimental data, a company provided, the values with lower and upper bounds that are defined in Table 1.

Two FE simulations are performed to replicate the radial and axial stiffness tests. If the simulated curves overlap the experimental counterpart, it means that the parameters of the constitutive equation having been selected could model this rubber-like material of rubber bushing accurately. To minimize the gap between simulated load-displacement curves and the experimental data, four subobjectives are proposed as follows.

Subobjective 1. The area enclosed by the simulated axial stiffness curve and abscissa axis is introduced as a response for this subobjective, while the experiment counterpart is applied as the target value.

Subobjective 2. The area enclosed by the simulated radial stiffness curve and abscissa axis is introduced as a response for this subobjective, while the experiment counterpart is applied as the target value.

Subobjective 3. The endpoint value on axial stiffness simulated curve is expected to approximate the experimental counterpart.

Subobjective 4. The endpoint value on radial stiffness simulated curve is expected to approximate the experimental counterpart.

Since the experimental and simulated curves are linear ranging from the minimum to the maximum value of the loads applied in this research, the above four subobjectives are able to make the simulated curves overlap the experimental 


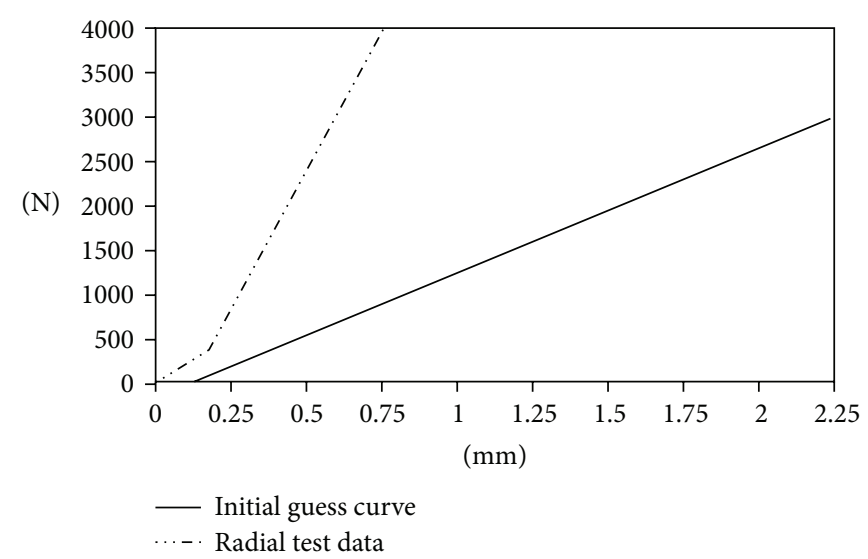

(a)

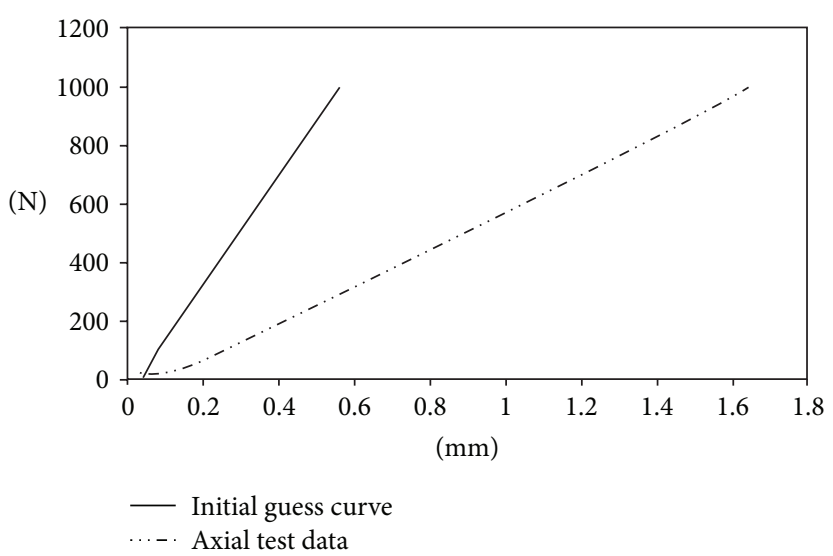

(b)

FIgURE 4: The initial load-displacement curves.

TABLE 2: The coefficient of material law (after optimized evaluation).

\begin{tabular}{lcc}
\hline Constitutive equation & $\mu_{i}$ & $\alpha_{i}$ \\
\hline & $\mu_{1}=1.273$ & $\alpha_{1}=0.985$ \\
Ogden $N=3$ & $\mu_{2}=2.58 e-04$ & $\alpha_{2}=11.495$ \\
& $\mu_{3}=5.8 e-06$ & $\alpha_{3}=-9.99$ \\
\hline
\end{tabular}

counterpart and then replicate the radial and axial stiffness tests.

In a usual optimization problem, only one design object should be defined, while in this specific case four subobjectives are involved in the identification process. A weighted equation is employed, therefore, to integrate the four goals mentioned above into one general objective:

$$
C_{W}=\sum_{i=1}^{4} \omega_{i} C_{i},
$$

where $C_{w}$ is the general objective as the weighted sum; $\omega_{i}$ are weights in this optimization procedure, $\omega_{1}=\omega_{2}=\omega_{3}=\omega_{4}=$ 1 ; and $C_{i}$ are the subobjective function.

Genetic algorithm [17] is introduced as the optimization method to find optimum value of the constitutive parameters of rubber-like material, due to its implicit parallelism and capability searching for the global optimum solution. The optimization procedure is carried out by FE optimization software. The iteration history plot will show the graph of the objectives. Figure 5 reveals the objectives history plot which converges to the specified values.

Having experienced 10 iterations, the result converges. Then it is extracted from the simulated model with parameters of constitutive model characterized simultaneously. The simulated versus experimental displacement-stress curvilinear relationship is shown in Figure 6, and a detailed parameters after the characterizing of the three-term Ogden law are provided in Table 2.

Figure 6 reveals good fit in both radial and axial stiffness with a slight relative errors involved which are acceptable in the engineering project. Consequently, the parameters of

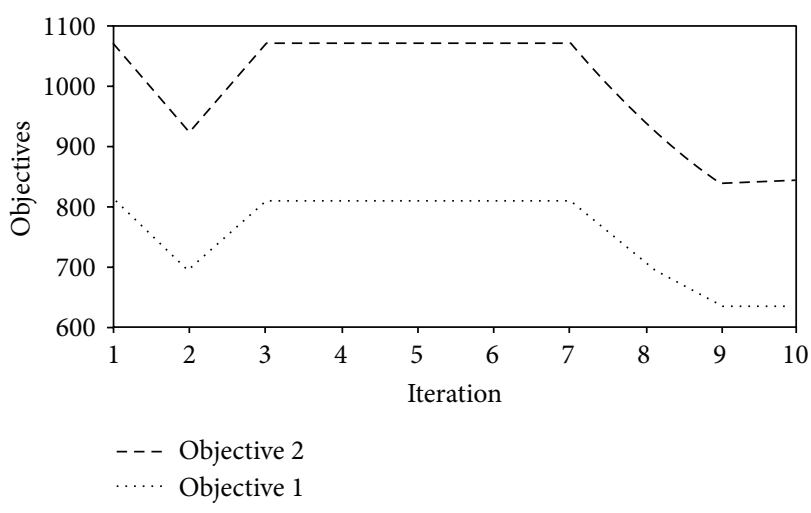

(a)

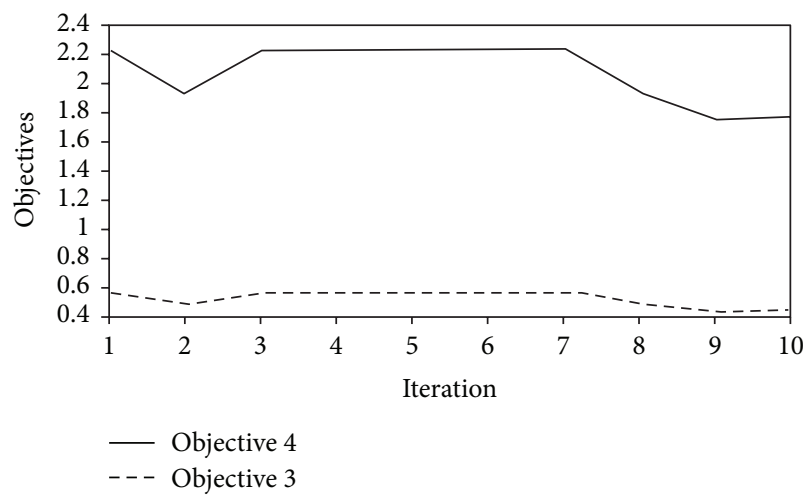

(b)

FIgURE 5: Histories of design variable.

constitutive model, obtained after final iteration, are proved validly to simulate the rotational stiffness.

\section{Rotational Stiffness Analysis}

Due to the limited amount of experimental appliances, the rotational stiffness tests are not conducted. Instead, they will 


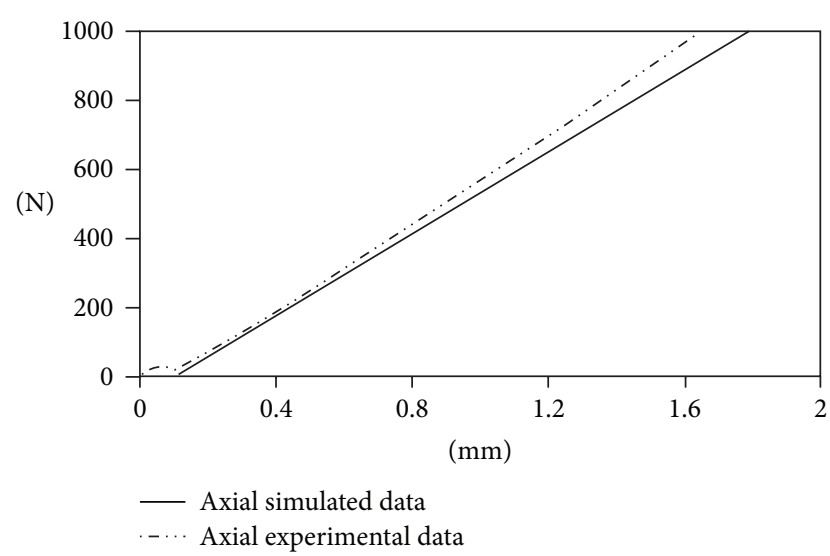

(a)

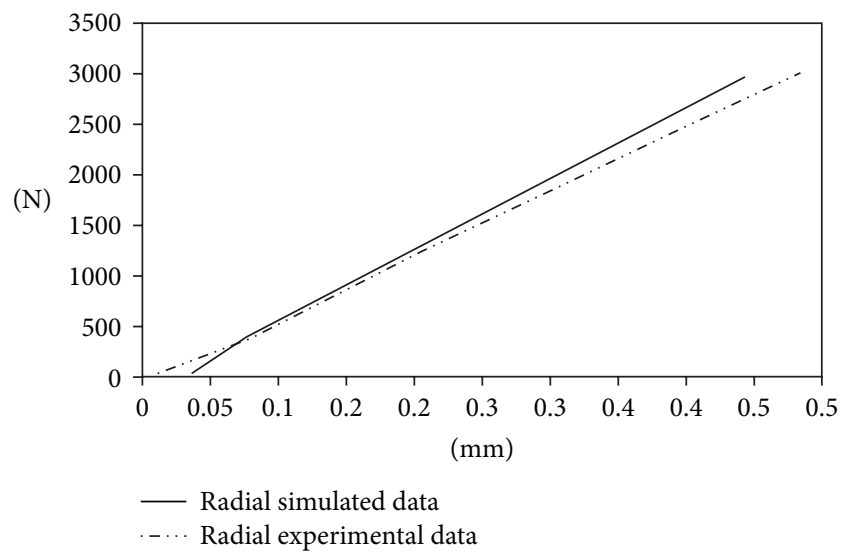

(b)

Figure 6: The load-displacement curves (after optimization).

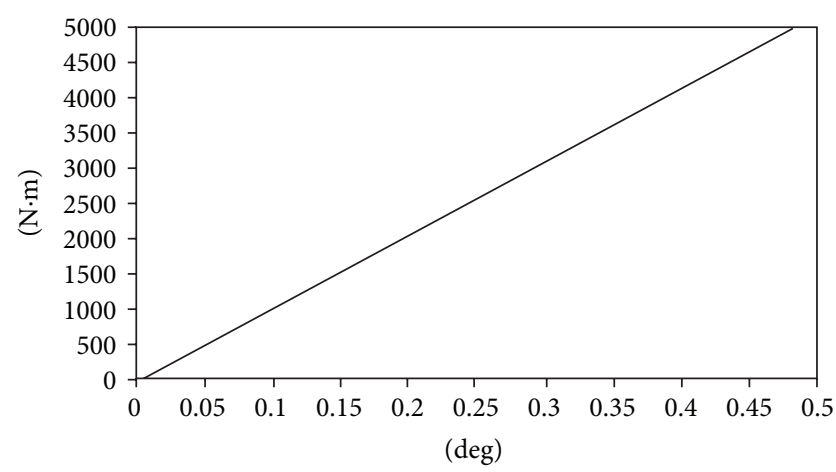

FIGURE 7: Rotational stiffness curve (around $y$ or $z$ direction).

be obtained through simulated software based on FE theory. The rotational stiffness information can be obtained by applying torques incrementally from $0 \mathrm{~N} \cdot \mathrm{mm}$ to $5000 \mathrm{~N} \cdot \mathrm{mm}$ around $x$ and $0 \mathrm{~N} \cdot \mathrm{mm}$ to $500 \mathrm{~N} \cdot \mathrm{mm}$ around $y(z)$ direction, respectively. The simulated torque-angle curves are provided in Figures 7 and 8.

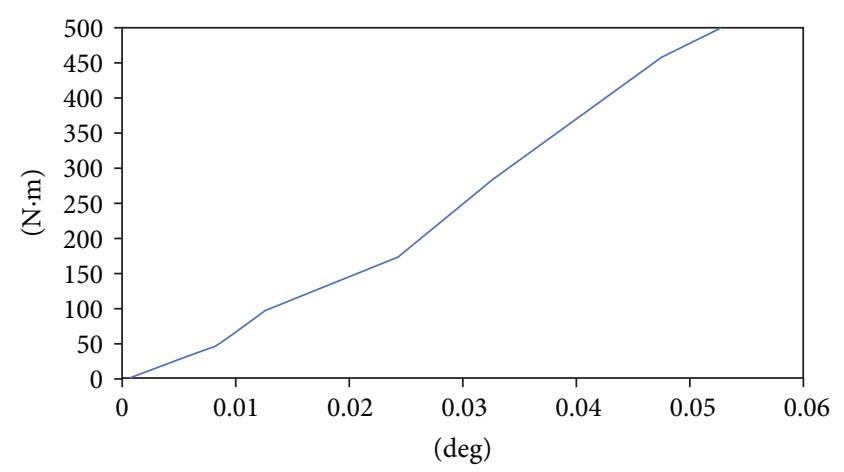

FIGURE 8: Rotational stiffness curve (around $x$ direction).

\section{Conclusions}

In this study, the parameters of the three-term Ogden law used for modeling hyperelastic material have been identified. A nonlinear simulation is performed to replicate experimental radial and axial stiffness test for a specific rubber bushing, while the parameters of the material law are determined to fit the experimental results. Comparing the experimental and simulated data, only petty errors are found which indicates that the parameters of the constitutive equation are valid for modeling the material of this rubber bushing.

Subsequently, an accurate model is established relying on the determinate parameter of the material law in order to evaluate two rotational stiffness curves which have not been obtained due to the lack of rotational test data. In this procedure, the complete information about stiffness of rubber bushing can be acquired.

This parameter identification method is proved to model the appropriate hyperelastic material for rubber bushing validly when material tensile tests data are not provided. Additionally, the whole information about rubber bushing stiffness can be established practically.

\section{Acknowledgment}

This work was funded by the 2012 open-ended foundation of Key Laboratory of Advanced Manufacturing Technology for Automobile Parts, Ministry of Education by Project of cstc2013yykfB0198.

\section{References}

[1] D. J. Charlton, J. Yang, and K. K. Teh, "Review of methods to characterize rubber elastic behavior for use in finite element analysis," Rubber Chemistry and Technology, vol. 67, no. 3, pp. 481-503, 1994.

[2] S. C. Chen, X. K. Tian, and W. M. Yan, "Modeling and analysis of laminated rubber bearings under axial tensile loading," Materials and Structures, 2013.

[3] N. Suphadon and J. J. C. Busfield, "Elastic behaviour of rubber cylinders under combined torsion and tension loading," Plastics, Rubber and Composites, vol. 38, no. 8, pp. 337-342, 2009.

[4] K.-J. Gong, Y.-T. Wei, and J.-X. Ye, "Constitutive parametric experiment of tire rubber hyperelastic laws with application," 
Gongcheng Lixue/Engineering Mechanics, vol. 26, no. 6, pp. 193198, 2009.

[5] M. Baker and A. Shrot, "Inverse parameter identification with finite element simulations using knowledge-based descriptors," Computational Materials Science, vol. 69, pp. 128-136, 2013.

[6] E. Florentin and G. Lubineau, "Identification of the parameters of an elastic material model using the constitutive equation gap method," Computational Mechanics, vol. 46, no. 4, pp. 521-531, 2010.

[7] P. Martins, R. Jorge, and A. Ferreira, "A comparative study of several material models for prediction of hyperelastic properties: application to silicone-rubber and soft tissues," Strain, vol. 42, no. 3, pp. 135-147, 2006.

[8] M. Mooney, "A theory of large elastic deformation," Journal of Applied Physics, vol. 11, no. 9, pp. 582-592, 1940.

[9] R. Rivlin, "Large elastic deformations of isotropic materials. iv: further developments of the general theory A," Philosophical Transactions of the Royal Society of London, p. 379, 1948.

[10] E. Twizell and R. Ogden, "Non-linear optimization of the material constants in Ogden's stress-deformation function for incompressible isotropic elastic materials," Journal of the Australian Mathematical Society, pp. 424-434, 1983.

[11] O. Yeoh, "Some forms of the strain energy function for rubber," Rubber Chemistry and Technology, vol. 66, no. 5, pp. 754-771, 1993.

[12] J. Martins, E. Pires, R. Salvado, and P. Dinis, "A numerical model of passive and active behavior of skeletal muscles," Computer Methods in Applied Mechanics and Engineering, vol. 151, no. 3-4, pp. 419-433, 1998.

[13] X.-F. Li and X.-X. Yang, "A review of elastic constitutive model for rubber materials," China Elastomerics, vol. 15, no. 1, pp. 50$58,2005$.

[14] A. Andrade-Campos, P. Pilvin, J. Simões, and F. Teixeira-Dias, Software Development for Inverse Determination of Constitutive Model Parameters, Software Engineering: New Research, Nova Science, New York, NY, USA, 2009.

[15] Hibbit.Karlsson \& Sorensen, ABAQUS Theory Manual Version 6.7, Hibbit.Karlsson \& Sorensen, Providence, RI, USA, 2007.

[16] "Mechanics and thermomechanics of rubberlike solids," G. Saccomandi and R. W. Ogden, Eds., Springer, New York, NY, USA, 2004.

[17] Z. Michalewicz, Genetic Algorithms Data Structures Evolution Programs, Springer, New York, NY, USA, 1996. 

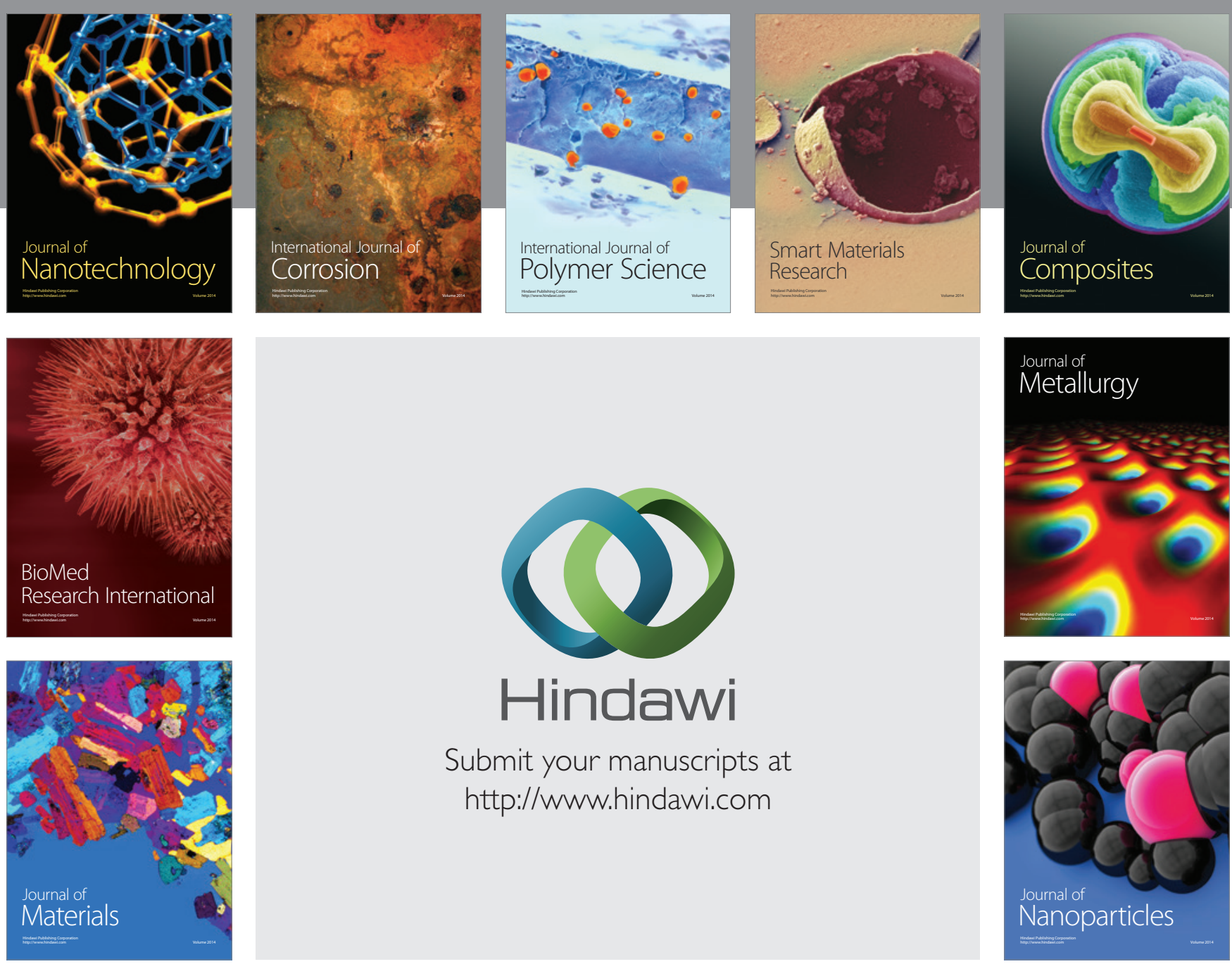

Submit your manuscripts at http://www.hindawi.com
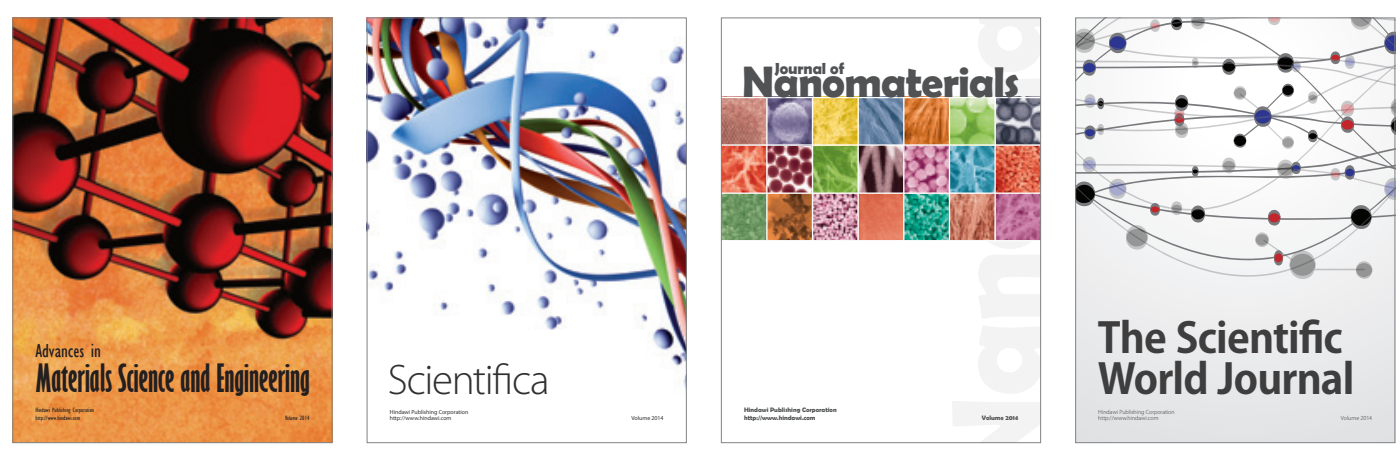

\section{The Scientific World Journal}
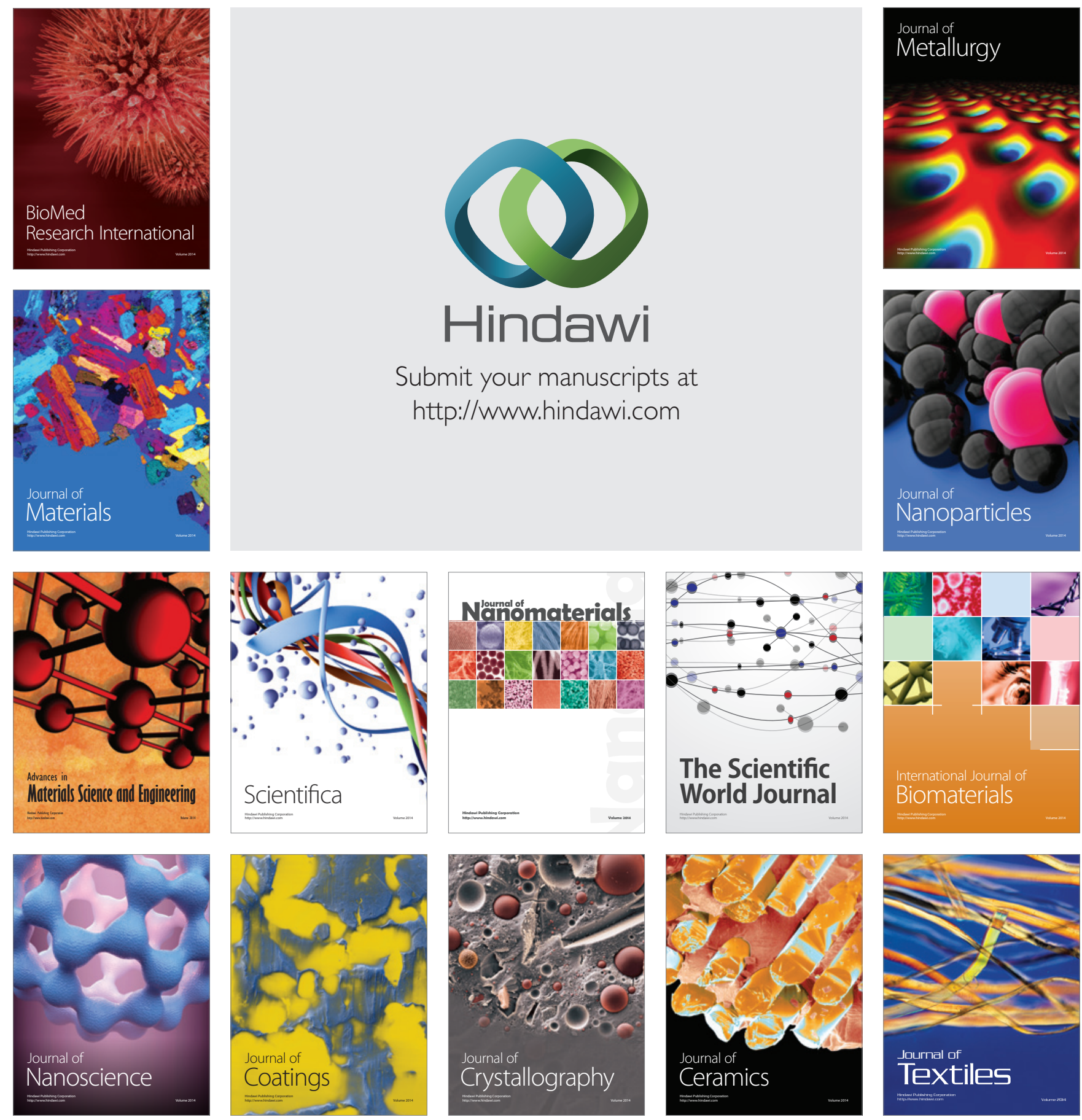